\title{
Effect of Wave Propagation in a Distributed Energy Absorption System for
}

\section{Trains}

\author{
Zhaoyang Ding ${ }^{1}$ Zhijun Zheng ${ }^{1, a}$ and Jilin $\mathrm{Yu}^{1}$ \\ ${ }^{1}$ CAS Key Laboratory of Mechanical Behavior and Design of Materials, Department of Modern Mechanics, University of Science and Technology of \\ China, Hefei 230026, China
}

\begin{abstract}
The influence of elastic wave propagation along the carriage in high-speed trains on the coordination of energy absorber components is analyzed in this paper. A simplified model is developed to simulate a "like to like" impact scenario of trains with distributed energy absorbing system. A rod made of elastic material is used to simulate a carriage, while the rigid-perfectly plastic-locking (R-PP-L) model is employed to describe the material of the energy absorber. The problem is simplified to a one-dimensional case. The response of absorption components during collision is divided into several stages and the corresponding governing equations of the impact response are obtained and numerically solved. A typical platform phenomenon of response in all stages is observed. The results of theoretical analysis are compared with those of finite element simulation and good agreement is achieved.
\end{abstract}

\section{Introduction}

The active and passive safety technology has received much attention with the development of high-speed trains. The passive safety technology is an efficient means of reducing losses in accidents when the active safety technology is failure. Several collision experiments of actual sized trains were implemented to examine the crashworthiness of train [1]. Recently, numerical simulation combined with theoretical analysis becomes the most commonly used and economical measure. The optimal methods for crashworthiness design [2,3] and the safety of passengers [4,5] have received much research interest. Multi-body dynamics analysis was widely used for train crash analysis [6, 7].

A train is a so long structure system that the length involved in the event increases with the passage of time and the impact load propagates from the front to the rear of a carriage in the form of elastic wave. Therefore, in order to develop the energy absorbing system, the effect of stress wave propagation along the train should be considered.

In this study, a simplified one-dimensional model, which takes the distribution of energy absorbing devices and the elasticity of the train carriages into consideration, is developed for a "like to like" impact scenario of trains. The governing equations of the impact response are obtained and numerically solved. The results are then compared with those of finite element (FE) simulation.

\section{A simplified model}

During collision, the axial action plays a decisive role both in load transfer and in compression failure behavior of energy absorbing components. Therefore, the train can be simplified to a one-dimensional model and can be investigated based on a one-dimensional stress wave theory, when neglecting the various influences of the detailed structures on the load transmission and energy absorption. Elastic rods are employed to represent the carriages, connected by plastic layers, which represent energy absorbing devices.

\footnotetext{
a Corresponding author: zjzheng@ustc.edu.cn
} 
As the plastic effect is more concerned rather than the elastic effect of energy absorbing device and the time scale of elastic wave propagation, the rigid-perfectly plastic-locking (R-PP-L) model [8] is employed to characterize the material response of energy absorbing device, as shown in Fig. 1, with the plateau stress and the densification strain corresponding to the mean crash force and the stroke efficiency, respectively. On the other hand, linear-elastic material is employed for carriage. As shown in Fig. 2, the simplified model of each carriage with energy absorbers is made up of an elastic compartment, and an energy absorption layer at the back-end, which is named as a distal layer. For simplicity, we put the energy absorbers between the nearby carriages together into the back end. For the head car, there is an extra energy absorbing layer at the front-end, which is named as a proximal layer.

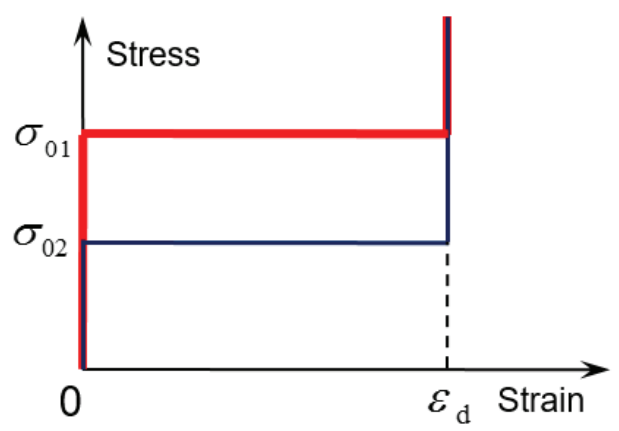

Figure. 1 The R-PP-L model.

To be noted, the energy-absorbing components in a distributed energy-absorbing system need to meet a certain distribution rule that the subsequent energy absorbing components should start to work before the one at the impact end is fully compacted. To meet this requirement, the plateau stress of the proximal layer $\left(\sigma_{01}\right)$ should be higher than that of the distal layer $\left(\sigma_{02}\right)$, as illustrated in Fig. 1.

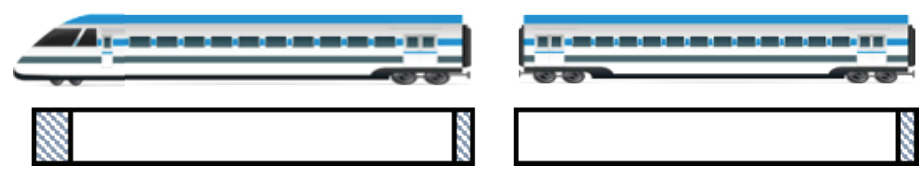

Figure. 2 A schematic diagram of the simplified model.

A collision scenario is analyzed in this study: a simplified model containing a head car and a carriage with an initial velocity impacting on a fixed rigid wall. This scenario simulates a "like to like" impact, in which the two trains with the same initial velocity and mass strike each other, as shown in Fig. 3.

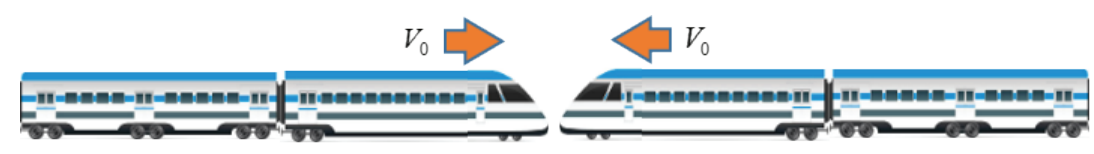

Like to like impact

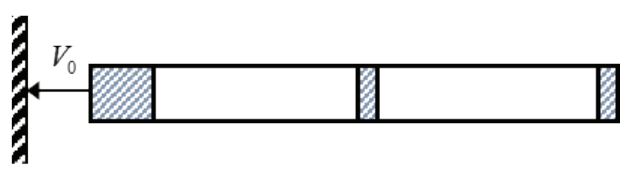

Figure. 3 The collision scenario of actual train and the corresponding simplified model.

\section{Theoretical derivation}

In this section, the collision scenario is investigated in detail and the governing equations of motion are analyzed based on Lagrangian coordinates. A schematic diagram of simplified model and the deformation process is presented in Fig. 4. 


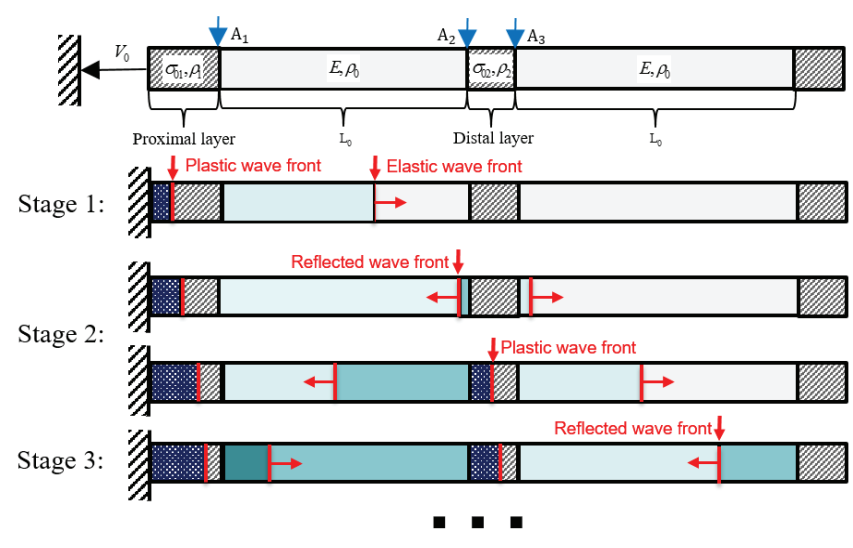

Figure. 4 A schematic diagram of the deformation process.

The parameters of the energy absorption layers used in the analysis of the simplified model are: the plateau stress $\sigma_{01}$ and $\sigma_{02}$ are $150 \mathrm{MPa}$ and $100 \mathrm{MPa}$, respectively; the density and length of the proximal layer are $\rho_{1}=600 \mathrm{~kg} / \mathrm{m}^{3}$ and $L_{1}=1 \mathrm{~m}$, and those of the distal layer are $\rho_{2}=400 \mathrm{~kg} / \mathrm{m}^{3}$ and $L_{2}=0.5 \mathrm{~m}$; the compaction strain is $\varepsilon_{\mathrm{d}}=0.8$ for both layers. The length of the elastic rod is $L_{0}=25$ $\mathrm{m}$, which has a Young's modulus $E=66000 \mathrm{MPa}$ and a density $\rho_{0}=2700 \mathrm{~kg} / \mathrm{m}^{3}$. The initial velocity of the simplified model of train is $V_{0}=-20 \mathrm{~m} / \mathrm{s}$.

Parameters to be solved are: the compaction length of the proximal layer $\Phi_{1}$ and that of the distal layer $\Phi_{2}$, the particle velocity $V_{R 1}$ at interface $A_{1}$ associated to the right traveling wave from interface $A_{1}$, particle velocity $v_{L 1}$ at interface $A_{2}$ associated to the left traveling wave from interface $A_{2}$, and particle velocity $v_{R 2}$ at interface $A_{3}$ associated to the right traveling wave from interface $A_{3}$. Other unknowns including the stress and particle velocity distribution can be obtained from these parameters.

Elastic wave propagation is the key for the load and impact energy transmissions between the proximal layer and the distal layer. So the analysis process can be divided into several stages by the characteristic time $t_{n}(n=1,2, \ldots)$ when elastic waves reach corresponding interfaces

$$
t_{n}=n L_{0} / C_{0}
$$

where $\mathrm{C} 0$ is the elastic wave speed, given by

$$
C_{0}=\sqrt{E / \rho_{0}} \text {. }
$$

\section{Stage $1\left(0<t<t_{1}\right)$}

When the front end hits the rigid wall, the proximal layer begins to collapse and a compaction wave starts propagating in the proximal layer. The stress ahead of the compaction wave front becomes $\sigma_{01}$ which pushes the rigid part of the proximal layer and decelerates it immediately. The stress on the interface $A_{1}$ rises and approaches $\sigma_{01}$. In the meanwhile, an elastic wave initiates and travels from interface $\mathrm{A}_{1}$ to interface $\mathrm{A}_{2}$ through the elastic rod at speed $C_{0}$.

For interface $\mathrm{A} 1$, the stress $\sigma_{\mathrm{A} 1}$ can be expressed by the particle velocity $\mathrm{v}_{\mathrm{R} 1}$. Using the compatibility relation of elastic wave and the initial conditions $\mathrm{v}_{\mathrm{R} 1}(0)=0$ and $\sigma_{\mathrm{A} 1}=0$, we have

$$
\sigma_{\mathrm{A} 1}=E v_{\mathrm{R} 1} / C_{0}
$$

By using the conservation of the mass of the proximal layer, the momentum theorem of the undeformed region of proximal layer and Eq. (3), the governing equations are obtained as

$$
\frac{\mathrm{d} \Phi_{1}^{(1)}}{\mathrm{d} t}=\frac{0-\left(V_{0}+v_{\mathrm{R} 1}^{(1)}\right)}{\varepsilon_{\mathrm{d}}}
$$

and

$$
\frac{\mathrm{d} v_{\mathrm{R} 1}^{(1)}}{\mathrm{d} t}=\frac{\sigma_{01}-E v_{\mathrm{R} 1}^{(1)} / C_{0}}{\rho_{1}\left(L_{1}-\Phi_{1}^{(1)}\right)}
$$

with the initial conditions $\Phi_{1}^{(1)}(0)=0$ and $v_{\mathrm{R} 1}^{(1)}(0)=0$, where superscript $(n)$ denotes the $n$-th stage of the response $(n=1,2, \ldots)$. 


\section{Stage $2\left(t_{1} \leq t<t_{2}\right)$}

When the elastic wave reaches interface $\mathrm{A}_{2}$, an elastic precursor wave travels through the distal layer with an infinite speed. Interface $\mathrm{A}_{3}$ starts to decelerate and an elastic wave propagates right. The response of interface $\mathrm{A}_{2}$ due to the right traveling wave is corresponding to that of interface $\mathrm{A}_{1}$ with only a time delay of

$$
\Delta t=L_{0} / C_{0} .
$$

The velocity and stress of interface $\mathrm{A}_{2}$ can be expressed as

$$
v_{\mathrm{A} 2}^{(2)}(t)=V_{0}+v_{\mathrm{R} 1}^{(1)}(t-\Delta t)+v_{\mathrm{L} 1}^{(2)}(t)
$$

and

$$
\sigma_{\mathrm{A} 2}^{(2)}(t)=\sigma_{\mathrm{R} 1}^{(1)}(t-\Delta t)+\sigma_{\mathrm{L} 1}^{(2)}(t)
$$

Originally, $\sigma_{\mathrm{A} 2}$ is lower than $\sigma_{02}$ and the distal layer remains undeformed. The equations of motion is given by the momentum theorem of distal layer

$$
\frac{\mathrm{d} v_{\mathrm{L} 1}^{(2)}}{\mathrm{d} t}=-\frac{2 E v_{\mathrm{L} 1}^{(2)}(t)}{C_{0} \rho_{2} L_{1}}-\frac{\mathrm{d} v_{\mathrm{R} 1}^{(1)}(t-\Delta t)}{\mathrm{d} t}
$$

with the initial condition $v_{\mathrm{L} 1}\left(t_{1}\right)=0$.

The time when $\sigma_{\mathrm{A} 2}$ reaches $\sigma_{02}$, denoted as $t_{\mathrm{c}}$, can be determined by Eqs. (8) and (9) and dealing with $\sigma_{\mathrm{L} 1}$ similarly as Eq. (3). Afterwards the distal layer begins to be crushed. The compaction stress on the collapsing wave front can be obtained by the momentum theorem of the infinitesimal there and the mass conservation in distal layer

$$
\sigma_{2}(t)=\sigma_{02}+\rho_{2}\left(v_{\mathrm{L} 1}^{(2)}(t)+v_{\mathrm{R} 1}^{(1)}(t-\Delta t)-v_{\mathrm{R} 2}^{(2)}(t)\right)^{2} / \varepsilon_{\mathrm{d}} .
$$

The equations of motion are obtained by the mass conservation, the momentum theorem of the deformed and undeformed regions of the distal layer

$$
\begin{gathered}
\frac{\mathrm{d} \Phi_{2}^{(2)}}{\mathrm{d} t}=\frac{v_{\mathrm{L} 1}^{(2)}(t)+v_{\mathrm{R} 1}^{(1)}(t-\Delta t)-v_{\mathrm{R} 2}^{(2)}(t)}{\varepsilon_{\mathrm{d}}} \\
\frac{\mathrm{d} v_{\mathrm{L} 1}^{(2)}}{\mathrm{d} t}=-\frac{\mathrm{d} v_{\mathrm{R} 1}^{(1)}(t-\Delta t)}{\mathrm{d} t}+\frac{1}{\rho_{2} \Phi_{2}^{(2)}(t)}\left[E\left(v_{\mathrm{R} 1}^{(1)}(t-\Delta t)-v_{\mathrm{L} 1}^{(2)}(t)\right) / C_{0}\right. \\
\left.-\sigma_{02}-\rho_{2}\left(v_{\mathrm{L} 1}^{(2)}(t)+v_{\mathrm{R} 1}^{(1)}(t-\Delta t)-v_{\mathrm{R} 2}^{(2)}(t)\right)^{2} / \varepsilon_{\mathrm{d}}\right] \\
\frac{\mathrm{d} v_{\mathrm{R} 2}^{(2)}}{\mathrm{d} t}=\frac{\sigma_{02}-E v_{\mathrm{R} 2}^{(2)} / C_{0}}{\rho_{2}\left(L_{1}-\Phi_{2}^{(2)}\right)}
\end{gathered}
$$

with the initial conditions $\Phi_{2}^{(2)}\left(t_{\mathrm{c}}\right)=0, v_{\mathrm{L} 1}^{(2)}\left(t_{\mathrm{c}}\right)=v_{\mathrm{R} 1}^{(1)}\left(t_{\mathrm{c}}-\Delta t\right)-C_{0} \sigma_{02} / E$ and $v_{\mathrm{R} 2}^{(2)}\left(t_{\mathrm{c}}\right)=2 v_{\mathrm{R} 1}^{(1)}\left(t_{\mathrm{c}}-\Delta t\right)-C_{0} \sigma_{02} / E$, where Eq. (10) is used.

\section{Stage $3\left(t_{2}<t<t_{3}\right)$}

The left-traveling wave reaches interface $\mathrm{A}_{1}$ at $t_{2}$, then the velocity response of interface $\mathrm{A}_{1}$ depends on the superposition of the left-traveling wave and a new right-traveling wave, which cause the equations of motion to be different from those of Stage 1 . We have

$$
\frac{\mathrm{d} \Phi_{1}^{(3)}}{\mathrm{d} t}=\frac{0-\left[V_{0}+\left(v_{\mathrm{R} 1}^{(3)}(t)+v_{\mathrm{L} 1}^{(2)}(t-\Delta t)\right)\right]}{\varepsilon_{\mathrm{d}}}
$$

and 


$$
\frac{\mathrm{d} v_{\mathrm{R} 1}^{(3)}}{\mathrm{d} t}=\frac{\sigma_{01}-E\left(v_{\mathrm{R} 1}^{(3)}(t)-v_{\mathrm{L} 1}^{(2)}(t-\Delta t)\right) / C_{0}}{\rho_{1}\left(L_{1}-\Phi_{1}^{(3)}(t)\right)}-\frac{\mathrm{d} v_{\mathrm{L} 1}^{(2)}(t-\Delta t)}{\mathrm{d} t}
$$

with the initial conditions $\Phi_{1}^{(3)}\left(t_{2}\right)=\Phi_{1}^{(1)}\left(t_{2}\right)$ and $v_{\mathrm{R} 1}^{(3)}\left(t_{2}\right)=v_{\mathrm{R} 1}^{(1)}\left(t_{2}\right)+v_{\mathrm{L} 1}^{(2)}\left(t_{2}-\Delta t\right)$.

For simplicity, only the theoretical derivations of the first three stages are demonstrated here. The derivations for more stages follow the similar rules and can be obtained.

\section{Results and discussion}

\subsection{Numerical solution of the theoretical model}

The fourth-order Runge-Kutta scheme is employed to solve the governing equations with the initial conditions given above. Typical velocity-time history and stress-time history at the three interfaces are shown in Fig. 5. As can be seen from this figure, the speed of the three interfaces can be described by several stages and keep approximately constant in each stage. For the definition of stage employed in this paper, interfacial velocities change once every two stages, which is equal to a time length of an elastic wave traveling back and forth between the two interfaces. The stress of the interfaces is almost equal to the corresponding plateau stress during collision and drops down afterwards.
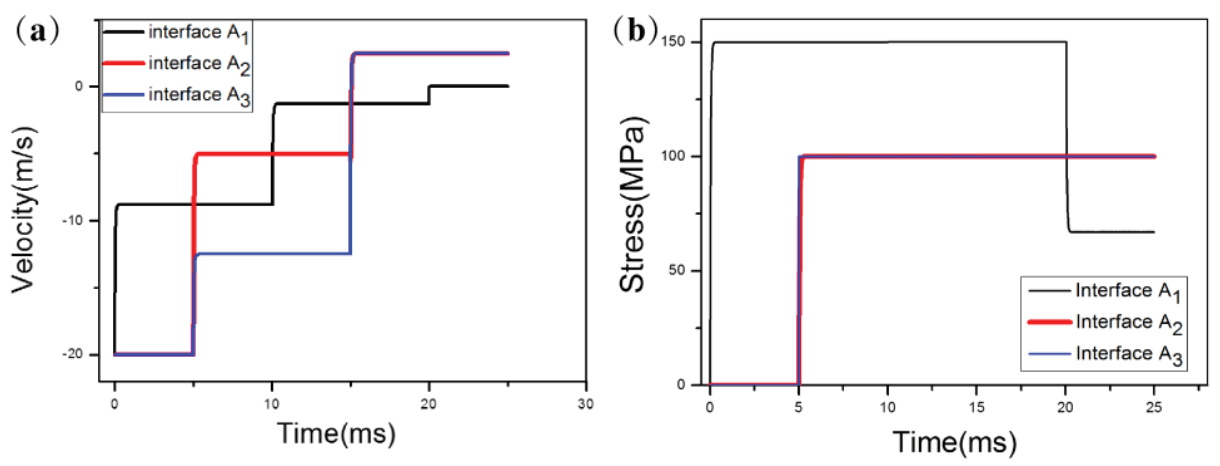

Figure. 5 Typical velocity-time history (a) and stress-time history (b) of interfaces $A_{1}, A_{2}$ and $A_{3}$.

\subsection{Comparison with finite element results}

Finite element method with ABAQUS/Explicit code is employed to verify the correctness of the theoretical analysis. The FE model is set up with the same parameters as the simplified model. Both the energy absorbing layers and the elastic connecting rods are modeled with C3D8 solid elements, of which the average length is set to be about $1 \mathrm{~mm}$ through a mesh sensitivity analysis. Crushable-foam-material and elastic-material are employed for the two layers and the elastic rods, respectively.

Local response of velocity and stress in the elastic rod is resulted from the superposition of two elastic waves, which can in turn verify the status of the two interfaces in each stage. Time histories of stress and velocity for selected Lagrangian locations in the first elastic rod are presented in Fig. 6. The thick black line is the result of the theoretical model for the midpoint. The thin and color lines are FE results for four selected positions, whose Lagrangian coordinates are $0, L_{0} / 4,3 L_{0} / 4$ and $L_{0}$. 

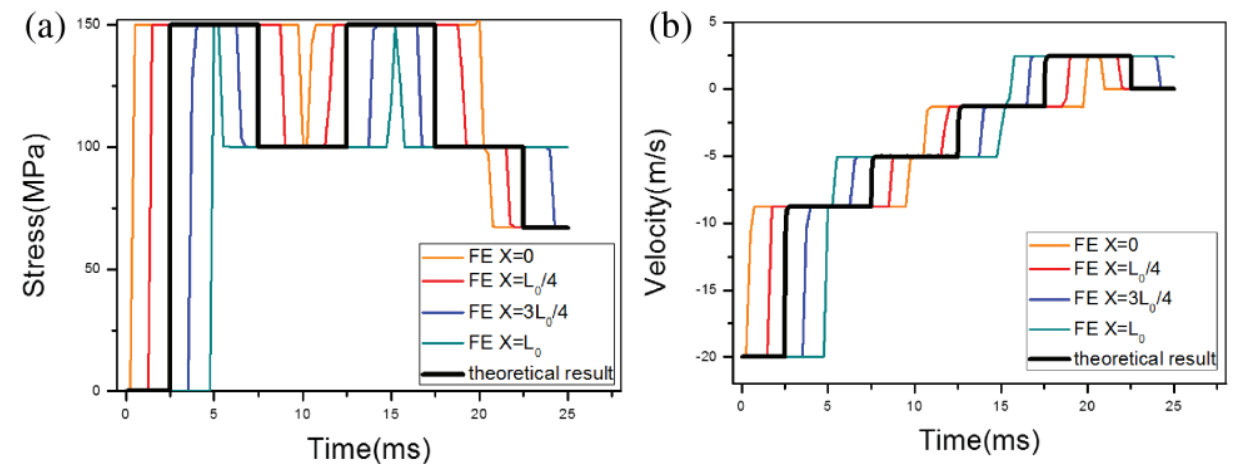

Figure. 6 Time histories of stress (a) and velocity (b) for selected Lagrange locations in the elastic rod.

Both the velocity and stress of the three interfaces are presented in Fig. 7. The theoretical and FE results are distinguished by lines without and with symbols, respectively.
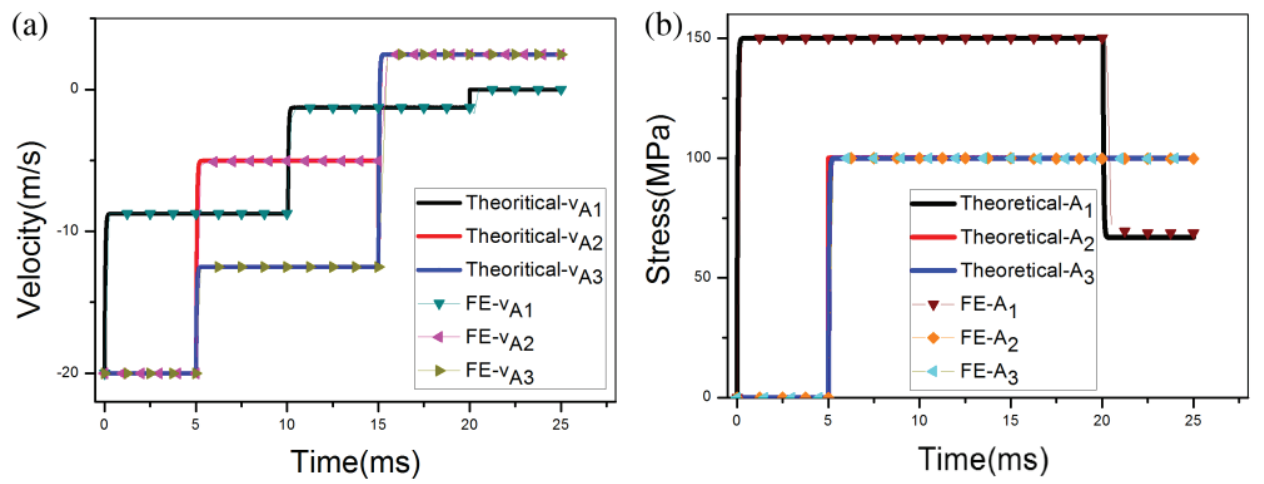

Figure. 7 Comparison between the results from theoretical analysis and FE simulations.

The observed agreement between the theoretical predictions and the FE results presented in Fig. 6 and Fig. 7 verifies the proposed theoretical approach. Minor difference of response time is result from that an infinite velocity of elastic wave front in two layers cannot be realized in the finite element simulation.

\section{Conclusions}

In this paper, a simplified model considering elastic wave effect is introduced to analyze the performance of the distributed energy absorbing system of trains. The governing equations for different stages of the response of the whole system in the course of collision are obtained and numerically solved. Typically segmented and platform typed phenomena of response are observed. The theoretical predictions are compared well with the finite element simulation results.

The proposed model is expected to be used for the analysis of the coordination of energy absorber components and preliminary design of the distributed energy absorption system for trains.

\section{Acknowledge}

The research reported herein is supported by the National Natural Science Foundation of China (Project No. 11372307), which is gratefully acknowledged.

\section{References}

1. P. Drazetic, R. Tassin, Y. Ravalard, et al, P I MECH ENG F-J RAI, 209, 11-17 (1995)

2. J. P. Dias, M. S. Pereira, COMPUT STRUCT, 82, 1371-1380 (2004)

3. G. J. Gao, H. Q. Tian, Int. J. Crashworthiness, 12, 21-28 (2007) 
4. J. W. Simons, S. W. Kirkpatrick, Int. J. Crashworthiness, 4, 121-132 (1998)

5. S. W. Kirkpatrick, M. Schroeder, J. W. Simons, Int. J. Crashworthiness, 6, 95-106 (2001)

6. J. A. C. Ambrósio, M. F. O. S. Pereira, J. P. Dias, NONLINEAR DYNAM, 10, 359-379 (1996)

7. H. S. Han, J. S. Koo, VEHICLE SYST DYN, 40, 435-450 (2003)

8. S. R. Reid, C. Peng, INT J IMPACT ENG, 19, 531-570 (1997) 